\title{
Mucosal Mast Cell Count Is Associated With Intestinal Permeability in Patients With Diarrhea Predominant Irritable Bowel Syndrome
}

\author{
Hyuk Lee, ${ }^{1}$ Jung Ho Park ${ }^{2 *}$ Dong II Park, ${ }^{2}$ Hong Joo Kim, ${ }^{2}$ Yong Kyun Cho, ${ }^{2}$ Chong II Sohn, ${ }^{2}$ Woo Kyu Jeon, ${ }^{2}$ Byung Ik Kim ${ }^{2}$ \\ and Seoung Wan Chae \\ ${ }^{1}$ Department of Internal Medicine and Institute of Gastroenterology, Yonsei University College of Medicine, Seoul, Korea; and Departments of \\ ${ }^{2}$ Internal Medicine and ${ }^{3}$ Pathology, Kangbuk Samsung Hospital, Sungkyunkwan University College of Medicine, Seoul, Korea
}

\section{Background/Aims}

Although mucosal mast cell tryptase is known to significantly increase intestinal permeability, the relationship between mucosal mast cells and intestinal permeability remains unclear. The objective of this study was to evaluate the correlation among intestinal permeability, tryptase activity and mucosal mast cell count.

\section{Methods}

Rectal biopsies from 16 patients with diarrhea-predominant irritable bowel syndrome (IBS-D) and 7 normal subjects were assessed for tryptase activity and macromolecular permeability using horseradish peroxidase in Ussing chambers. In addition, mucosal mast cell levels were immunohistochemically quantified via image analysis.

\section{Results}

Rectal biopsy of tissues from IBS-D patients showed significantly increased permeability compared with those from normal controls $\left(0.644 \pm 0.08\right.$ and $\left.0.06 \pm 0.00 \mathrm{ng} / 2 \mathrm{hr} / \mathrm{mm}^{2}, P<0.01\right)$. Tryptase activity was also substantially higher in rectal biopsy samples from IBS-D patients than those from normal controls $(0.86 \pm 0.18$ and $0.28 \pm 0.04 \mathrm{mU} / \mathrm{mg}$ protein, $P<0.05)$. Mucosal mast cell counts were not significantly different between the 2 groups $(P>0.05)$. However, correlation analysis revealed that only mucosal mast cell count was significantly correlated with intestinal permeability in IBS-D patients $(r=0.558$, $P<0.05)$.

\section{Conclusions}

This study demonstrated a positive correlation between the number of mucosal mast cells and intestinal permeability, suggesting that mucosal mast cells play an important role for increased intestinal permeability in patients with IBS-D.

\section{(J Neurogastroenterol Motil 2013;19:244-250)}

Key Words

Irritable bowel syndrome; Mast cell, Permeability; Tryptase

Received: October 11, 2012 Revised: December 17, 2012 Accepted: December 18, 2012

(c) This is an Open Access article distributed under the terms of the Creative Commons Attribution Non-Commercial License (http://creativecommons. org/licenses/by-nc/3.0) which permits unrestricted non-commercial use, distribution, and reproduction in any medium, provided the original work is properly cited.

*Correspondence: Jung Ho Park, MD

Department of Medicine, Sungkyunkwan University School of Medicine, Kangbuk Samsung Hospital, 29, Saemunan-ro, Jongno-gu, Seoul 110-746, Korea

Tel: +82-2-2001-2059, Fax: +82-2-2001-2485, E-mail: pjho3@hotmail.com

Financial support: None.

Conflicts of interest: None.

Author contributions: Jung Ho Park planned this study. Hyuk Lee, Jung Ho Park and Seoung Wan Chae conducted study. Hyuk Lee wrote the paper. Dong IL Park, Hong Joo Kim, Yong Kyun Cho, Chong IL Sohn, Woo Kyu Jeon and Byung Ik Kim contributed to revise and draft the manuscript. 


\section{Introduction}

Abnormal intestinal permeability has been implicated in the pathogenesis of many intestinal diseases, including irritable bowel syndrome (IBS). Symptoms of post-infectious IBS are associated with a subtle increase in intestinal permeability. ${ }^{1}$ Patients with diarrhea predominant IBS (IBS-D) have been shown to have increased small intestinal permeability compared to post-infectious IBS patients and controls. ${ }^{2}$ These findings suggest that an abnormal intestinal barrier facilitates enhanced antigen exposure that may activate the intestinal immune system and induce IBS symptoms such as abdominal pain.

Although the mechanism for increased intestinal permeability in IBS is still unclear, mast cells seem to play an important role. Recent studies have demonstrated that chronic stress increased the number and activity of mucosal mast cells and induced mass cell-mediated alterations in epithelial function. ${ }^{3-5}$ Furthermore, increased numbers of CD3, CD25 lymphocytes and mast cells have been detected in the colonic mucosa of IBS patients. ${ }^{6-8}$ Finally, it has been shown that IBS-D patients have increased rectal permeability responsive to mast cell tryptase.

In our previous study, ${ }^{9}$ we demonstrated that mucosal mast cell tryptase plays an important role in the increased rectal permeability in IBS. However, the relationship between mucosal mast cells and intestinal permeability remains unclear. Therefore, the goal of the present study is to evaluate the correlation among intestinal permeability, tryptase activity and mucosal mast cell count.

\section{Materials and Methods}

\section{Subjects}

Biopsy specimens of the rectum were obtained during routine colonoscopies of 16 patients with IBS-D and 7 healthy controls at the Kangbuk Samsung Hospital. All the IBS-D patients exhibited symptoms that fulfilled the Rome-II criteria. Participants in the control group had macroscopically and histologically normal colonic mucosa, no persistent bowel symptoms, no organic or functional bowel disease, and no history of chronic medical disease. None of the IBS-D patients had known histories of abdominal surgery, inflammatory bowel disease, or post-infectious IBS. Informed written consent was provided by all patients, and this study was approved by the hospital's local ethics committee.
Biopsy forceps with an opening diameter of 6 mm (FB-25K-1; Olympus, Tokyo, Japan) were used for the procedures. In most cases, 2 biopsies were taken from the rectum of IBS-D patients and controls.

\section{Staining and Quantification of Mucosal Mast Cells}

\section{Staining for mucosal mast cells}

Rectal mucosal mast cells were stained using a monoclonal antibody against the human mast cell protease tryptase. Biopsy specimens were fixed in $10 \%$ neutral buffered formalin for 24 hours. For the immunohistochemistry, paraffin-embedded specimens were cut with a microtome at $4 \mu \mathrm{m}$ thickness. Just before staining, slides were deparaffinized in xylene and rehydrated in graded alcohol solutions. After dewaxing, tissue sections were incubated with $0.5 \%$ hydrogen peroxide in methanol at $22^{\circ} \mathrm{C}$ for 10 minutes and then washed with running tap water for 15 minutes. Sections were treated with $0.1 \%$ trypsin (Sigma, Poole, UK) mixed in $0.1 \%$ calcium chloride ( $\mathrm{pH} 7.8$ ) for 10 minutes at $37^{\circ} \mathrm{C}$. Nonspecific binding of protein was blocked by incubation in normal rabbit serum diluted 1:5 in Tris-buffered saline (TBS; $\mathrm{pH}$ 7.6) for 15 minutes.

The slides were then incubated for 18 hours at $4^{\circ} \mathrm{C}$ with the monoclonal antibody AA1 (anti-human mast cell protease tryptase; DAKO M7052, Dako Ltd., Cambridge, UK), washed in TBS for 7 minutes, and reincubated for 30 minutes with biotinylated rabbit anti-mouse IgG (Amersham, Buckinghamshire, UK). After the slides were washed again in TBS for 7 minutes, finally they were incubated with streptavidin-biotin complex conjugated with horseradish peroxidase (HRP; DAKO k0377; Dako) for 30 minutes.

The slides were developed in diaminobenzidine-hydrogen peroxide substrate (Sigma) for 10 minutes, and washed under running tap water for 5 minutes. Sections were then counterstained with hematoxylin followed by dehydration and clearance. And then they were mounted in DPX mountant (BDH Prolabo; VWR International Ltd., Leicestershire, UK).

\section{Quantification of mucosal mast cells}

Mucosal mast cells were counted under light microscopy at $\times 400$ magnification by an expert pathologist. Immunohistochemically positive-stained mast cells were counted in five consecutive non-overlapping microscopic fields ( 3 sections $\times 2$ biopsy samples) with areas of $0.24 \mathrm{~mm}^{2}$. The pathologist did not know histological section's group of origin. 


\section{Measurement of Intestinal Permeability}

The biopsy tissues were mounted in modified Ussing chambers. After mounting, each half chamber was filled with 2 $\mathrm{mL}$ Krebs-Ringer bicarbonate (KRB) solution, bathing both the mucosal and serosal sides of the specimen. Both sides were gassed with $95 \% \mathrm{O}_{2}$ and $5 \% \mathrm{CO}_{2}$ and the temperature was kept at $37^{\circ} \mathrm{C}$ by heat block. After a 30-minute equilibration period, the $\mathrm{KRB}$ in the chamber of mucosal side was replaced with $\mathrm{KRB}$ containing $\mathrm{HRP}$ at a final concentration of $0.4 \mathrm{mg} / \mathrm{mL}$, and the $\mathrm{KRB}$ on the serosal side was substituted for fresh KRB. A $0.3 \mathrm{~mL}$ sample was collected and replaced with $0.3 \mathrm{~mL} \mathrm{KRB}$ on the serosal side every 30 minutes, for a total of 120 minutes. Samples from the serosal chamber were analyzed enzymatically using a modified Worthington method with o-dianisidine dihydrochloride (OPD; Sigma Chemical Co., St Louis, MO, USA) as the substrate. Samples of $50 \mu \mathrm{L}$ were relocated to microtitre plates. Then, 100 $\mu \mathrm{L}$ of OPD Working Solution (Stable Peroxide Buffer diluted 1:10 in OPD solution) was transferred to each well, and the plate

A

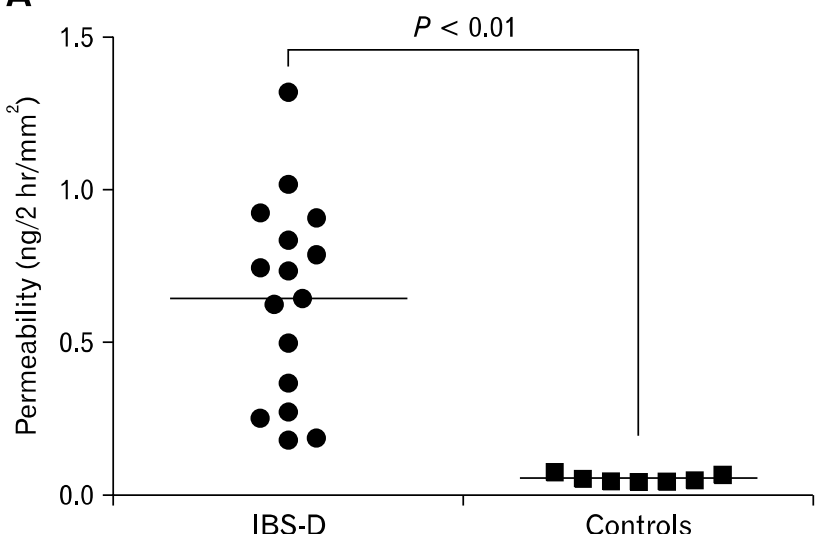

C

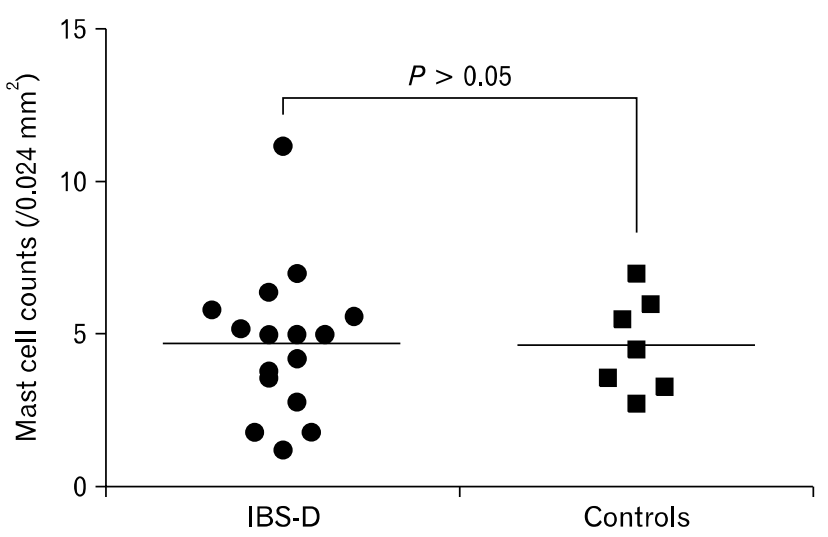

was incubated in a shaker at $300 \mathrm{rpm}$ at room temperature. After 30 minutes, $100 \mu \mathrm{L}$ of $2.5 \mathrm{M}$ sulfuric acid was applied, and 10 minutes later, the absorbance of the discolorized reaction product was estimated at a wavelength of $490 \mathrm{~nm}$ using a microplate reader (Model 680; Bio-Rad Laboratories, Inc., Hercules, USA). All samples were run in duplicate and measured in reference to a standard curve. HRP flux was demonstrated as $\mathrm{ng} / 2 \mathrm{hr} / \mathrm{mm}^{2}$ during steady-state permeation in 30 minutes interval.

\section{Tryptase Activity}

Ten microliter of the supernatant from a tissue extract were applied to $200 \mu \mathrm{L}$ of buffer $(50 \mathrm{mmol} / \mathrm{L}$ Tris- $\mathrm{HCl}, \mathrm{pH} 7.6 ; 120$ $\mathrm{mmol} / \mathrm{L} \mathrm{NaCl} ; 20 \mathrm{~g} / \mathrm{L}$ heparin) containing $0.5 \mathrm{mmol} / \mathrm{L}$ mast cell tryptase substrate (tosyl-glycine-proline-arginine-p-nitroanilide) and then they were incubated at room temperature for 17 hours. Substrate cleavage was measured using a microplate reader at an absorbance of $415 \mathrm{~nm}$ and normalized to the protein concentration of the supernatant. Concentrations were measured automatically against a standard curve. A specific mast cell tryptase
B

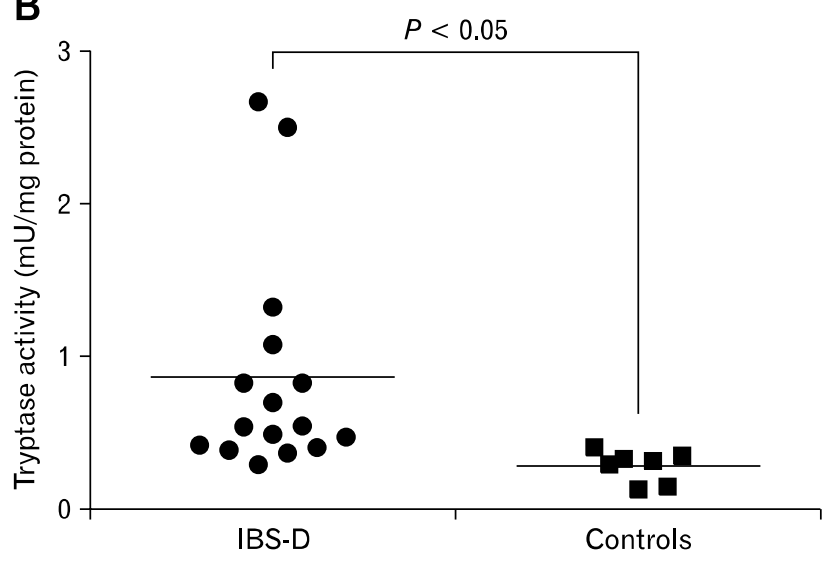

Figure 1. Comparison of intestinal permeability, tryptase activity and mucosal mast cell count between IBS-D patients $(\mathrm{n}=16)$ and controls $(\mathrm{n}$ $=7$ ). Intestinal permeability (A) and tryptase activity (B) of rectal biopsy tissues from IBS-D patients were significantly increased compared with biopsy tissues from normal controls. (C) However, mucosal mast cell counts were not significantly different between the 2 groups. 
inhibitor (nafamostat mesilate) was used to confirm assay specificity.

\section{Statistical Methods}

All data are expressed as mean \pm standard deviation (SD). Two-tailed Student's $t$ test was used for comparison of statistical differences in permeability, mucosal mast cell count and tryptase activity between normal subjects and IBS-D patients. Correlations were analyzed by Pearson's correlation. The alpha level of significance was set at $P<0.05$. All analyses were performed using SPSS (version 11.5.0; SPSS Inc., Chicago, IL, USA).

\section{Results}

\section{Patient Characteristics}

Sixteen patients with IBS-D (10 females, aged 24-66 years,

A

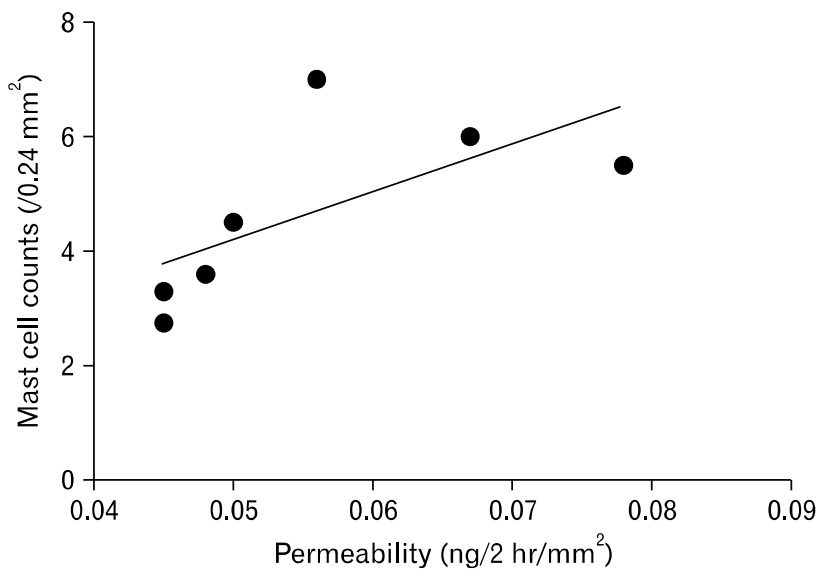

C

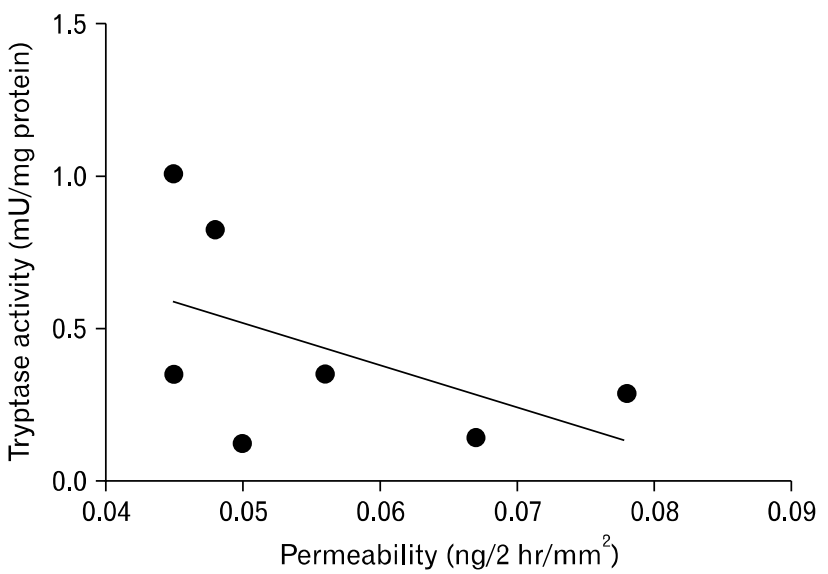

mean 54.6 years) and 7 health controls (3 females, aged 38-64 years, mean 49.0 years) were included. There was no significant difference in age and sex between 2 groups $(P>0.05$, respectively). The median symptom duration was 65 months (range 7-360 months).

\section{Comparison of Intestinal Permeability,} Tryptase Activity and Mucosal Mast Cell Count Between Diarrhea Predominant Irritable Bowel Syndrome Patients and Controls

Rectal biopsy samples from IBS-D patients showed significantly increased permeability compared with those from normal controls $\left(0.64 \pm 0.08\right.$ and $0.06 \pm 0.00 \mathrm{ng} / 2 \mathrm{hr} / \mathrm{mm}^{2}, P<$ $0.01)$ (Fig. 1). Tryptase activity was also remarkably higher in rectal biopsy samples from IBS-D patients than those from normal controls $(0.86 \pm 0.18$ and $0.28 \pm 0.04 \mathrm{mU} / \mathrm{mg}$ protein, $P<$ $0.05)$. However, mucosal mast cell counts were not significantly

B

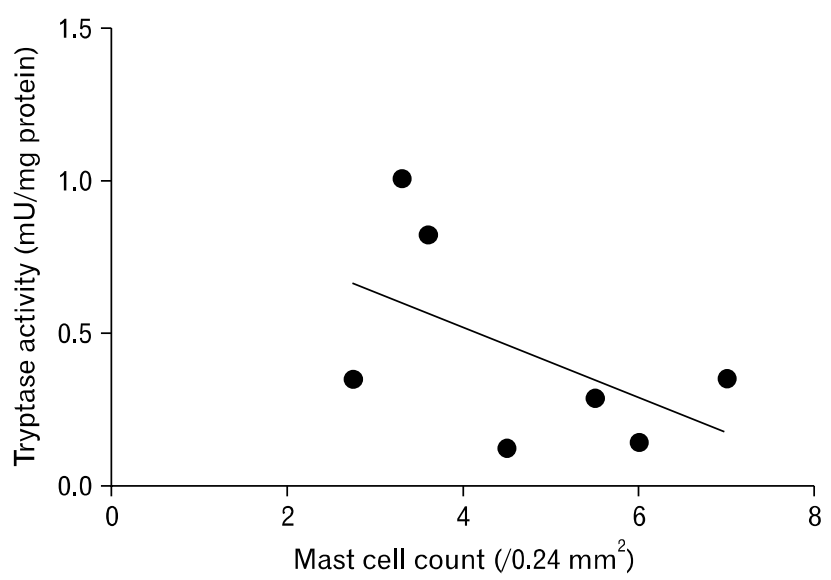

Figure 2. Correlation among mucosal mast cell count, tryptase activity and intestinal permeability in normal controls. There was no significant relationship between mucosal mast cell count and permeability (A), tryptase activity and mucosal mast cell count (B), and tryptase activity and permeability $(\mathrm{C})(P>0.05, \mathrm{n}=7$, respectively). 
different between the 2 groups $(4.71 \pm 0.60$ and $4.66 \pm 0.59$ number/0.24 $\left.\mathrm{mm}^{2}, P>0.05\right)$.

\section{Correlation Among Mucosal Mast Cell Count, Tryptase Activity and Intestinal Permeability in Normal Controls}

There was no remarkable relationship among intestinal permeability, tryptase activity and mucosal mast cell count in the control group $(P>0.05)$ (Fig. 2).

\section{Correlation Among Mucosal Mast Cell Count, Tryptase Activity and Intestinal Permeability in Diarrhea Predominant Irritable Bowel Syndrome Patients}

A significant positive correlation between mucosal mast cell count and intestinal permeability was observed $(r=0.558, P<$ $0.05)$. However, there were no notable relationships between intestinal permeability and tryptase activity $(r=-0.347, P>0.05)$ or mucosal mast cell count and tryptase activity $(r=-0.448, P>$

A

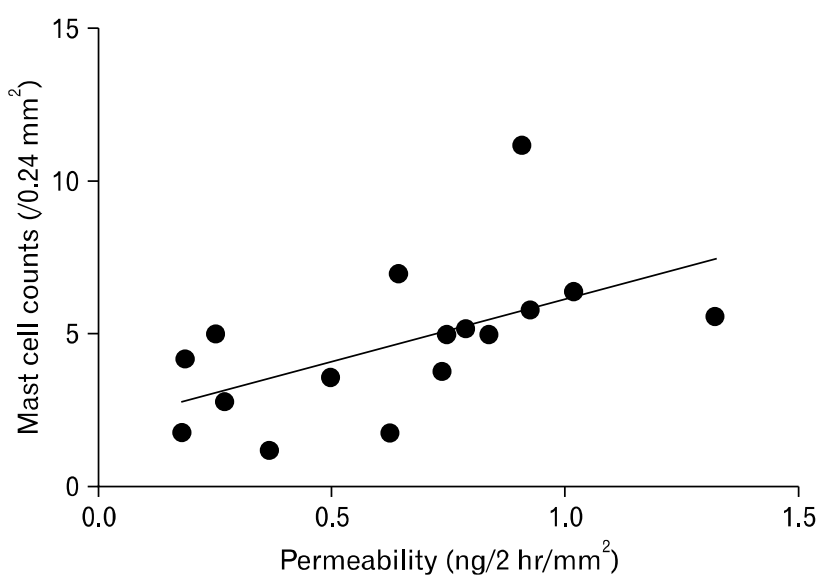

C

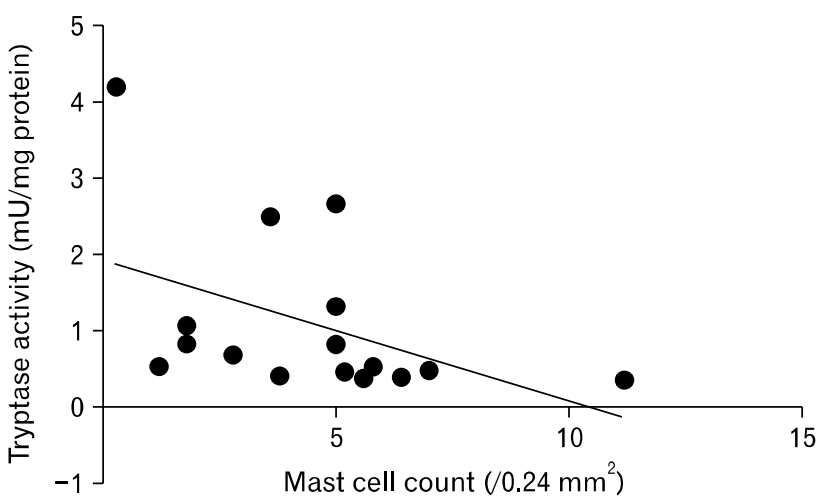

$0.05)$ (Fig. 3).

\section{Discussion}

This study demonstrated that mucosal mast cell count is strongly associated with intestinal permeability. Even though there was no significant difference in mucosal mast cell count between IBS-D patients and normal controls, intestinal permeability and tryptase activity were significantly higher in biopsy tissues from IBS-D patients.

There is growing evidence that low grade mucosal inflammation plays an important role in the development of IBS, ${ }^{10}$ with particular attention on mucosal mast cells. Several reports have suggested that IBS patients have a significantly increased number of mucosal mast cells compared with normal controls. ${ }^{6,11}$ However, other studies have failed to show differences in mucosal mast cell count between IBS patients and controls. ${ }^{12,13}$ In this study, the mucosal mast cell counts of IBS patients were similar to those of normal controls, while intestinal permeability dif-

B

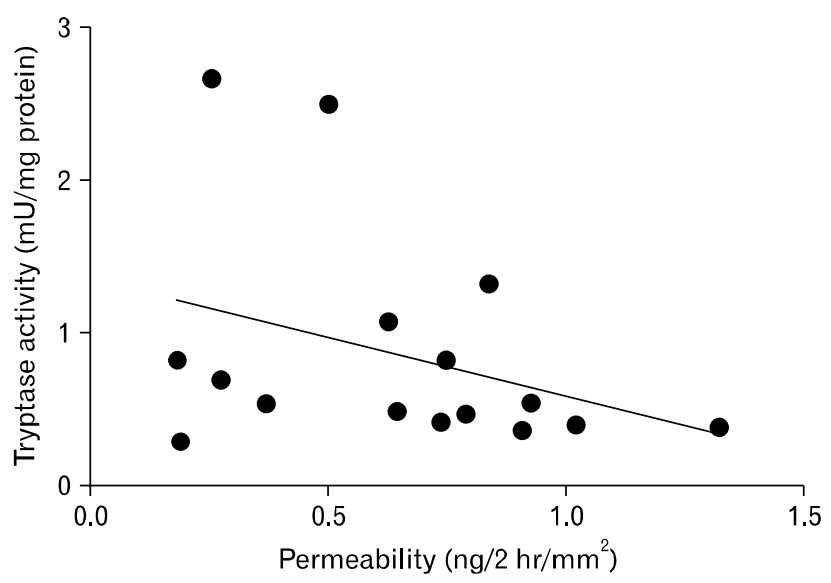

Figure 3. Correlation among intestinal permeability, mucosal mast cell count and tryptase activity. (A) A significant correlation was found between intestinal permeability and mucosal mast cell count $(r=$ 0.558, $P<0.05)$. However, there were no notable relationships between intestinal permeability and tryptase activity (B) or mucosal mast cell count and tryptase activity $(\mathrm{C})(P>0.05, \mathrm{n}=16$, respectively). 
fered between the 2 groups. These results are not consistent with our previous findings of both higher numbers of mucosal mast cells ${ }^{6}$ and increased permeability in IBS patients.

Although small sample size and differences in symptom severity between IBS-D patients could lead to inconsistent results, a possible explanation for this discrepancy in mucosal mast cell count and intestinal permeability between the 2 groups is that activated mast cells may be associated with increased intestinal permeability rather than simply an increase in mucosal mast cell count. Compared to biopsy tissues from normal controls, those from IBS patients showed significantly higher tryptase activity, which is an indicator of mast cell activation in various diseases. ${ }^{14,15}$ Therefore, despite having similar numbers of mucosal mast cells, the increased number of activated mast cells may induce increased intestinal permeability in IBS patients.

One interesting finding from this study was the positive correlation between mucosal mast cell count and intestinal permeability in IBS patients. In our earlier study, mucosal mast cell count was associated with visceral hypersensitivity in IBS-D patients. ${ }^{6}$ Similarly, other studies have reported that increased intestinal permeability in IBS patients may lead to IBS symptoms and visceral hypersensitivity. ${ }^{16,17}$ Taken together, these data and our results from the present study support the hypothesis that activated mast cells may be responsible for the enhanced intestinal permeability and sensitivity observed in IBS patients.

Tryptase is the most abundant mediator stored in mast cell granules and has been used as a marker for mast cell activation. ${ }^{18}$ Its activation of protease-activated receptor 2 (PAR-2) on enterocytes causes changes in the organization of tight junction and permits the passage of macromolecules across the epithelial barrier. ${ }^{19,20}$ Although biopsy tissues from IBS patients in the present study exhibited high levels of tryptase activity and increased permeability, tryptase activity did not correlate with intestinal permeability or mucosal mast cell count. This result likely indicates that enhanced tryptase activity is only partially responsible for the increased permeability, and that other mast cell mediators or neural regulators may participate in the control of intestinal permeability. ${ }^{3,21}$ Further investigation is needed to identify and verify other mast cell factors that may control intestinal permeability.

In conclusion, rectal tissues from IBS-D patients showed increased permeability and tryptase activity compared to those from normal controls. In addition, mucosal mast cell number positively correlated with intestinal permeability. These findings will be important in understanding the pathogenesis of increased intestinal permeability in IBS patients.

\section{References}

1. Marshall JK, Thabane M, Garg AX. Intestinal permeability in patients with irritable bowel syndrome after a waterborne outbreak of acute gastroenteritis in Walkerton, Ontario. Aliment Pharmacol Ther 2004;20:1317-1322.

2. Dunlop SP, Hebden J, Campbell E, et al. Abnormal intestinal permeability in subgroups of diarrhea-predominant irritable bowel syndromes. Am J Gastroenterol 2006;101:1288-1294.

3. Santos J, Yang PC, Söderholm JD, Benjamin M, Perdue MH. Role of mast cells in chronic stress induced colonic epithelial barrier dysfunction in the rat. Gut 2001;48:630-636.

4. Demaude J, Salvador-Cartier C, Fioramonti J, Ferrier L, Bueno L. Phenotypic changes in colonocytes following acute stress or activation of mast cells in mice: implications for delayed epithelial barrier dysfunction. Gut 2006;55:655-661.

5. Jacob C, Yang PC, Darmoul D, et al. Mast cell tryptase controls paracellular permeability of the intestine. Role of protease-activated receptor 2 and beta-arrestins. J Biol Chem 2005;280:31936-31948.

6. Park JH, Rhee PL, Kim HS, et al. Mucosal mast cell counts correlate with visceral hypersensitivity in patients with diarrhea predominant irritable bowel syndrome. J Gastroenterol Hepatol 2006;21: 71-78.

7. Chadwick VS, Chen W, Shu D, et al. Activation of the mucosal immune system in irritable bowel syndrome. Gastroenterology 2002; 122:1778-1783.

8. Barbara G, Stanghellini V, De Giorgio R, et al. Activated mast cells in proximity to colonic nerves correlate with abdominal pain in irritable bowel syndrome. Gastroenterology 2004;126:693-702.

9. Lee JW, Park JH, Park DI, et al. Subjects with diarrhea-predominant IBS have increased rectal permeability responsive to tryptase. Dig Dis Sci 2010;5 5:2922-2928.

10. Dunlop SP, Jenkins D, Spiller RC. Distinctive clinical, psychological, and histological features of postinfective irritable bowel syndrome. Am J gastroenterol 2003;98:1578-1583.

11. O'Sullivan M, Clayton N, Breslin NP, et al. Increased mast cells in the irritable bowel syndrome. Neurogastroenterol Motil 2000;12: 449-457.

12. Talley NJ, Butterfield JH. Mast cell infiltration and degranulation in colonic mucosa in the irritable bowel syndrome. Am J gastroenterol 1996;91:1675-1676.

13. Spiller RC, Jenkins D, Thornley JP, et al. Increased rectal mucosal enteroendocrine cells, $\mathrm{T}$ lymphocytes, and increased gut permeability following acute Campylobacter enteritis and in post-dysenteric irritable bowel syndrome. Gut 2000;47:804-811.

14. Schwartz LB, Metcalfe DD, Miller JS, Earl H, Sullivan T. Tryptase levels as an indicator of mast-cell activation in systemic anaphylaxis and mastocytosis. N Eng J Med 1987;316:1622-1626.

15. Butrus SI, Ochsner KI, Abelson MB, Schwartz LB. The level of tryptase in human tears. An indicator of activation of conjunctival mast cells. Ophthalmology 1990;97:1678-1683.

16. Zhou Q, Zhang B, Verne GN. Intestinal membrane permeability and hypersensitivity in the irritable bowel syndrome. Pain 2009;146: 
41-46.

17. Ait-Belgnaoui A, Bradesi S, Fioramonti J, Theodorou V, Bueno L. Acute stress-induced hypersensitivity to colonic distension depends upon increase in paracellular permeability: role of myosin light chain kinase. Pain 2005;113:141-147.

18. Miller HR, Pemberton AD. Tissue-specific expression of mast cell granule serine proteinases and their role in inflammation in the lung and gut. Immunology 2002;105:375-390.

19. Cenac N, Coelho AM, Nguyen C, et al. Induction of intestinal in- flammation in mouse by activation of proteinase-activated receptor-2. Am J pathol 2002;161:1903-1915.

20. Chin AC, Vergnolle N, MacNaughton WK, Wallace JL, Hollenberg MD, Buret AG. Proteinase-activated receptor 1 activation induces epithelial apoptosis and increases intestinal permeability. Proc Nat Acad Sci USA 2003;100:11104-11109.

21. Santos J, Saunders PR, Hanssen NP, et al. Corticotropin-releasing hormone mimics stress-induced colonic epithelial pathophysiology in the rat. Am J Physiol 1999;277(2 Pt 1):G391-G399. 